\title{
Original
}

\section{Conocimientos, actitudes y prácticas sobre donación y trasplante de córneas en comunidades rurales y urbanas de Honduras}

\section{Knowledge, attitudes and practices about corneal donation and transplantation in rural and urban communities in Honduras.}

Ana Carolina Valeriano Rosales ${ }^{1 *}$ Yolanda María Fernández Muñoz ${ }^{1}$ Dennis Josué Quiroz Zelaya ${ }^{1}$ Allison Suyapa Young Banegas ${ }^{1}$ María Fernanda Martínez Zavala ${ }^{1}$ Laura Patricia Delgadillo Guilbert ${ }^{1}$ María del Milagro Estrada Fúnez ${ }^{1}$

${ }^{1}$ Universidad Católica de Honduras

*Correspondencia a: anavaleriano15@hotmail.com

Palabras clave: Córnea, Trasplante, Cadáver, Actitud donante, Honduras.

Keywords: Cornea, Transplantation, Cadaver, Donor attitude, Honduras.

\section{Historia del artículo}

Recepción: 06 noviembre 2020

Aprobación: 25 mayo 2021

DOI: https://doi.org/10.5377/ rcfh.v7i1.11758

Citar como: Valeriano Rosales AC, Fernández Muñoz YM, Quiroz Zelaya DJ, Young Banegas AS, Martínez Zavala MF, Delgadillo Guilbert LP, Estrada Fúnez MdM. Conocimientos, actitudes y prácticas sobre donación y trasplante de córneas en comunidades rurales y urbanas de Honduras Rev. cienc. forenses Honduras. 2021; 7(1): 23-39. DOI: https://doi.org/10.5377/ rcfh.v7i1.11758

Conflicto de interés: ninguno https://orcid.org/0000-0002-0866-1730

https://orcid.org/0000-0003-2393-110X

https://orcid.org/0000-0002-5413-0367

https://orcid.org/0000-0002-0435-6835

https://orcid.org/0000-0001-5884-823X

https://orcid.org/0000-0001-6075-4793

https://orcid.org/0000-0001-5093-1362

\section{RESUMEN}

Introducción: aproximadamente 600 personas urgen de trasplante de córnea en Honduras. La mayor dificultad para este procedimiento es que no se cuenta con banco de ojo en el país. Además, los estudios de conocimiento sobre donación y trasplante, son escasos. Objetivo: conocer las actitudes y prácticas de la población rural versus la urbana sobre la donación y trasplante de córnea, según conocimientos y factores sociodemográficos, con el fin de diseñar un plan de acción para concientizar la importancia de una conducta donante positiva para el beneficio del paciente con ceguera corneal. Metodología: estudio descriptivo realizado entre mayo y septiembre del 2019 en Comayagua, Intibucá y Francisco Morazán. Con una muestra de 350 personas por departamento, para un total de 1050 participantes mayores de 18 años y de ambos sexos. Se realizó entrevista por investigadores, bajo la escala de Likert, previamente validado. Variables agrupadas en demográficas, socio personales, laborales, conocimiento, creencias y aptitudes. Se analizaron los datos mediante razón de probabilidades y productos cruzados. Los resultados se expresaron en porcentajes, frecuencias y odd ratio. Resultados: $74.86 \%$ manifestó una actitud donante positiva, el $67.55 \%$ donaría a cualquier persona que lo necesitase,
Rev. cienc. forenses Honduras, Volumen 7, N 1, Año 2021

Open Access
(c)Autor(es).
URL: http://www.bvs.hn/RCFH/html5/ CC-BY-NC 4.0 internacional 
48\% conocía acerca de las características requeridas para el donante y. menos del $10 \%$ sabía qué es la córnea. Los factores negativos para la donación son la procedencia rural, la parentela y el desempleo en Intibucá y motivos religiosos en el área rural de Comayagua y población urbana de Francisco Morazán. Conclusiones: Es importante superar las limitaciones impuestas por la desinformación, para ello es necesario impulsar una cultura de donación a través de la creación de estrategias comunicativas de educación, con el fin de mejorar la salud visual y la demanda poblacional

\section{ABSTRACT}

Justification: approximately 600 people require corneal transplantation in Honduras. The greatest difficulty for this procedure is that there is no eye bank in the country. Furthermore, knowledge studies on donation and transplantation are scarce. Objective: to know the attitudes and practices of the rural versus the urban population regarding corneal donation and transplantation, according to knowledge and sociodemographic factors, in order to design an action plan to raise awareness of the importance of positive donor behavior for the benefit of the patient with corneal blindness. Methodology: descriptive study carried out between May and September
2019 in Comayagua, Intibucá and Francisco Morazán. With a sample of 350 people per department, for a total of 1050 participants over 18 years of age and of both sexes. An interview was conducted by researchers, under the Likert scale, previously validated. Variables grouped in demographic, socio-personal, work, knowledge, beliefs and skills. The data were analyzed using odds ratios and cross products. The results were expressed in percentages, frequencies and odd ratio. Results: $74.86 \%$ expressed a positive donor attitude, $67.55 \%$ would donate to anyone who needed it, $48 \%$ know about the characteristics required for the donor and. less than $10 \%$ know what the cornea is. The negative factors for the donation are rural origin, relatives and unemployment in Intibucá and religious reasons in the rural area of Comayagua and urban population of Francisco Morazán. Conclusions: it's important to overcome the limitations imposed by misinformation, for this it is necessary to promote a culture of donation through the creation of educational communication strategies, in order to improve visual health and population demand.

\section{INTRODUCCIÓN}

En las Américas en el año 2016 se realizaron más de 53.000 trasplantes $^{1}$, siendo el trasplante de córnea, el realizado con más frecuencia en 
todo el mundo ${ }^{2}$. La tasa por millón de personas aumentó 6,8\% respecto al 2015, según el Observatorio Global sobre Donación y Trasplante (GODT) de la Organización Mundial de la Salud (OMS), correspondiendo al trasplante de córnea cerca de 40.000 trasplantes por año en esta región ${ }^{1}$.

Diversos factores influyen en la cultura de donación como ser la educación, el ingreso económico, el tratamiento del tema con la familia y el conocimiento de lugares que brindan información sobre donación ${ }^{3}$, estos están relacionados con el grado de desarrollo de la población y el contexto cultural específico de cada sociedad influyendo en algunas, la posición religiosa y la autoridad y la jerarquía de los padres en la familia ${ }^{4}$, por lo que es importante que previo a promover la donación cadavérica en nuestro país, se exploren cuáles son las creencias y actitudes de la población hondureña al respecto.

Al igual que lo realizado en otros países, España ${ }^{5}$, Perú ${ }^{6}$ y México ${ }^{7}$; en Honduras, en el año 2017 se realizó un estudio transversal, descriptivo, mediante un muestreo aleatorio simple de 300 individuos que encontró que el $70 \%$ de los entrevistados tenían una intención positiva hacia la donación ${ }^{8}$. Sin embargo, debido a que este estudio se enfocó en población urbana, es importante conocer y comparar cuáles son los conocimientos y actitudes de la población rural versus la urbana, sobre el trasplante y donación, específicamente de córneas, (por ser este el más factible de implementar a corto plazo en nuestro país), a fin que se diseñe un plan de acción eficaz y concientizar a la población sobre la importancia de tener una conducta donante positiva para el beneficio del paciente con ceguera corneal; considerando además que el país dispone de una ley para trasplante de órganos y tejidos, que faculta legalmente el proceso y se cuenta además con recursos humanos capacitados ${ }^{9-11}$.

\section{METODOLOGÍA}

Se realizó un estudio descriptivo, tipo encuesta CAP (Conocimientos, Actitudes y Prácticas) en los departamentos de Francisco Morazán, Comayagua e Intibucá durante el periodo de mayo a septiembre de 2019. Se utilizó como marco referencial de cada municipio los datos del XVII censo de población y vivienda del año 2013 y registro Catastral del 2010- 2019 ${ }^{12-16}$.

Se seleccionaron los barrios y comunidades mediante un generador de números aleatorios, se tomaron dos barrios o colonias del sector urbano y dos del sector rural, así:

1.-En el departamento de Francisco Morazán: los municipios de Monte Redondo, San Juancito 
(Distrito Central) y La Ermita, Talanga, como zona rural y barrio El Manchen y Buenos Aires como urbanos.

2.- En el departamento de Comayagua: en el municipio de Siguatepeque, la Aldea El Porvenir y Aldea Balibrea, como zona rural, y el barrio Suyapita y El Parnaso, como zona urbana.

3.-En el departamento de Intibucá: en el municipio de Jesús de Otoro se tomó como zona rural Maye y en Santo Domingo como zona urbana, barrio Santa Cruz y colonia San Miguel. Dentro de cada barrio o colonia se enumeraron las casas y se seleccionaron aleatoriamente y dentro de estas a los individuos que cumplían con los criterios de inclusión.

El universo del estudio fue la población total mayor de 18 años de cada municipio siendo un absoluto de 84,636 personas según el Instituto Nacional de Estadísticas (INE) del año 2012. Se tomó una muestra, aleatoria por conglomerados bi-etápicos de 350 personas en cada departamento con un nivel de confianza del $95 \%$ y un margen de error del $5 \%$, para una muestra total de 1050 individuos.

Los criterios de inclusión fueron: población mayor de 18 años y de ambos sexos en las comunidades seleccionadas. Tomando en cuenta el $50 \%$ de la población evaluada en las zonas declaradas como urbanas y $50 \%$ en zonas declaradas como rurales de cada municipio.

La recopilación de datos se obtuvo mediante la aplicación de un instrumento, previamente validado. La sección de conocimiento bajo la escala de Likert, con variables agrupadas en demográficas: municipio, procedencia, variables socio personales: sexo, edad, escolaridad y parentela (hijos o no), filiación religiosa; variables laborales: situación laboral, y nivel socioeconómico según la escala Necesidades Básicas Insatisfechas (NBI) ${ }^{17}$, variables de conocimiento y variables de actitudes y creencias sobre la donación y trasplante de córnea.

El conocimiento de la población encuestada se evaluó mediante cinco parámetros:

1. ¿Sabe si en Honduras hay o existe donación de órganos?.

2. ¿Qué es la córnea?.

3. ¿Conoce si en Honduras se realiza trasplante de córnea?.

4. Existencia del banco de córnea en Honduras.

5. Características del donante.

La actitud hacia la donación se evaluó mediante cinco parámetros:

1. Disposición a donar la córnea al fallecer. 
2.-Razones por las cuales no donaría la córnea al fallecer (religión, no hay remuneración económica, mutilación del cuerpo, desinformación del procedimiento y otras razones).

3. ¿A quién aceptarían donarle su cornea al fallecer? (familiar, amigo, desconocido).

4. Disposición a formar parte de una lista de posibles donantes en Honduras.

5. Disposición a portar un carnet que lo acredite como donador de órganos.

Los datos recopilados se analizaron con el programa Epi Info versión 7.2.3.1 año 2019 ${ }^{18}$, mediante razón de probabilidades y productos cruzados. Utilizando el valor de $p$ igual a 0.05, el intervalo de confianza y el valor de $\mathrm{Chi}^{2}$ para identificar la relación de variables.

Los resultados se expresaron en porcentajes, frecuencias y odd ratio.

A cada persona encuestada se le solicitó el llenado del consentimiento informado en el cual se le explicó la naturaleza de la investigación, los objetivos y propósitos, se les garantizó la confidencialidad de la información y el uso meramente para fines investigativos $y$ académicos.

\section{RESULTADOS}

En la muestra analizada, tanto en el área urbana, como rural de los tres departamentos el grupo etario mayormente representado fue el comprendido entre 18-27 años (32.0\%), del sexo femenino (67.43\%), con hijos (79.90\%), pobre o en pobreza extrema (53.25\%), que laboraba (54.10\%), católicos $(52,86 \%)$; y con escolaridad de primaria completa o menos (50.86\%). Los datos demográficos, socioculturales y laborales están descritos y estratificados según área de procedencia en el cuadro 1.

\section{Actitud ante la donación de córnea}

En relación a la disposición a donar, el 74.86\% (786/1050) manifestó una actitud positiva al respecto; de los cuales el $67.55 \%$ donaría su córnea a cualquier persona que lo necesitase y el $32.44 \%$ únicamente a familiares y amigos. De estos el $59 \%$ estaba de acuerdo en formar parte de una lista de posibles donantes y $48.5 \%$ en portar un carnet que lo acredite como tal, se observó menor disposición de adquirir un documento que le respalde como donante en el área rural. $(P=0.00001)$.

El análisis intra-departamental y por área de procedencia indica que:

1.-En Comayagua, el $87.14 \%$ de la población tiene una actitud donante positiva (rural: 
44.04\%, urbana: 43.1\%), en Intibucá el 65.15\% (rural: $26.3 \%$, urbana: $38.9 \%$ ) y en Francisco Morazán el 72.29\% (rural: 38.3\%, urbana: 34\%). En el departamento de Intibucá se observó un mayor porcentaje de aceptación en el área urbana en contraste con un mayor porcentaje de rechazo en el área rural. $(P=0.00005$, $\left.X^{2}=24.2905\right)$. Véase cuadro 2.

En Comayagua la principal causa de rechazo fue la idea de mutilación del cuerpo con el 40\%; en Francisco Morazán e Intibucá "razones personales privadas". Se observaron diferencias entre las razones de rechazo según área de procedencia; en el apartado "por miedo a que me retiren los órganos antes de estar realmente muerto" con mayor porcentaje en el área rural de Comayagua e Intibucá. Solo en Intibucá el apartado "por motivos religiosos" fue preponderante en el área rural. Ver cuadro 3.

\section{Variables socio-personales}

Independientemente del área de procedencia (rural o urbana), se encontraron diferencias significativas en todos los departamentos en la intención a donar, relacionadas a la edad $(O R=$ $0.08, I C=0,02-0.28$ ); se observó que a partir de los 58 años la actitud donante es negativa en contraste con la población de menor edad. En Francisco Morazán, el sexo se observó como un factor significativo para la donación $(O R=0.65$,
$I C=0.40-1.06) ; \quad$ los hombres tuvieron una percepción negativa a esta.

En relación a la situación laboral y parentela (hijos o no) solamente se encontró relevancia significativa en el departamento de Intibucá. Las personas desempleadas en el área rural $(p=0.0164, O R=1.95, I C=1.24-3.08) ; y$ el tener descendencia en el área urbana y rural $(p=0.0344, \quad O R=0.33, I C=0.17-0.64) ;$ son un factor negativo hacia la donación de córnea.

La religión es un factor independiente en el departamento de Intibucá sin distinción de área de procedencia, sin embargo, en Comayagua, en el área rural, los practicantes Testigo de Jehová y adventistas están en contra de la donación. ( $\left.X^{2}=26.83, p=0.0001\right)$ observando el mismo resultado en Francisco Morazán en el área urbana. $\left(X^{2}=39.51, p=0.00001\right)$.

\section{Variables de conocimiento sobre donación de}

\section{Córnea}

El 95\% ( $n=999)$ de los participantes conoce que aún no se cuenta con un banco de ojos en el país, el $48 \%(n=505)$ conoce acerca de las características del donante y el $10 \%$ sabe qué es la córnea y que ya se realizan trasplantes de esta en el país.

Se encontraron diferencias en el nivel de 


\begin{tabular}{|c|c|c|c|c|c|c|c|c|c|}
\hline \multicolumn{10}{|c|}{ Cuadro 1. Características socio demográficas, socio personales y laborales } \\
\hline & & \multicolumn{2}{|c|}{ Comayagua } & \multicolumn{2}{|c|}{ Intibucá } & \multicolumn{2}{|c|}{ Francisco Morazán } & \multirow[b]{2}{*}{ Total } & \multirow[b]{2}{*}{ Porcentaje } \\
\hline & & Rural & Urbano & Rural & Urbano & Rural & Urbano & & \\
\hline & Mujer & 120 & 128 & 124 & 109 & 126 & 101 & 708 & $67.43 \%$ \\
\hline Sexo & Hombre & 55 & 47 & 51 & 66 & 49 & 74 & 342 & $32.57 \%$ \\
\hline \multirow{8}{*}{ Edad } & $18-27$ & 56 & 60 & 64 & 55 & 52 & 49 & 336 & $32.00 \%$ \\
\hline & $28-37$ & 48 & 43 & 44 & 50 & 43 & 33 & 261 & $24.86 \%$ \\
\hline & $38-47$ & 28 & 37 & 29 & 25 & 25 & 28 & 172 & $16.38 \%$ \\
\hline & $48-57$ & 27 & 29 & 16 & 20 & 17 & 30 & 139 & $13.24 \%$ \\
\hline & $58-67$ & 10 & 5 & 13 & 12 & 21 & 14 & 75 & $7.14 \%$ \\
\hline & $68-77$ & 3 & 1 & 7 & 8 & 10 & 18 & 47 & $4.48 \%$ \\
\hline & $78-87$ & 3 & 0 & 1 & 5 & 7 & 3 & 19 & $1.81 \%$ \\
\hline & $88-97$ & 0 & 0 & 1 & 0 & 0 & 0 & 1 & $0.10 \%$ \\
\hline \multirow{8}{*}{ Escolaridad } & $\begin{array}{l}\text { Analfabeta } \\
\text { Sabe leer y }\end{array}$ & 13 & 12 & 13 & 7 & 6 & 0 & 51 & $4.86 \%$ \\
\hline & escribir & 7 & 9 & 3 & 0 & 4 & 4 & 27 & $2.57 \%$ \\
\hline & $\begin{array}{l}\text { Primaria } \\
\text { Incompleta }\end{array}$ & 36 & 19 & 50 & 33 & 50 & 20 & 208 & $19.81 \%$ \\
\hline & $\begin{array}{l}\text { Primaria } \\
\text { Completa }\end{array}$ & 41 & 37 & 61 & 40 & 54 & 15 & 248 & $23.62 \%$ \\
\hline & $\begin{array}{l}\text { Secundaria } \\
\text { Incompleta }\end{array}$ & 27 & 20 & 27 & 25 & 25 & 13 & 137 & $13.05 \%$ \\
\hline & Secundaria & 21 & & 21 & $2 \sigma$ & 20 & ك & ונד & ס \\
\hline & Completa & 44 & 47 & 16 & 45 & 21 & 46 & 219 & $20.86 \%$ \\
\hline & Universidad & 7 & 31 & 5 & 25 & 15 & 77 & 160 & $15.24 \%$ \\
\hline \multirow{4}{*}{$\begin{array}{c}\text { Situación } \\
\text { laboral }\end{array}$} & Trabaja & 87 & 107 & 81 & 96 & 76 & 121 & 568 & $54.10 \%$ \\
\hline & Desempleado & 83 & 64 & 88 & 68 & 97 & 18 & 418 & $39.81 \%$ \\
\hline & Estudiante & 4 & 1 & 6 & 10 & 1 & 21 & 43 & $4.10 \%$ \\
\hline & Jubilado & 1 & 3 & 0 & 1 & 1 & 15 & 21 & $2.00 \%$ \\
\hline \multirow{2}{*}{ Tiene hijos } & Si & 140 & 142 & 148 & 129 & 160 & 120 & 839 & $79.90 \%$ \\
\hline & No & 35 & 33 & 27 & 46 & 15 & 55 & 211 & $20.10 \%$ \\
\hline \multirow{3}{*}{$\begin{array}{l}\text { Nivel socio } \\
\text { económico } \\
*\end{array}$} & No pobre & 76 & 93 & 72 & 67 & 76 & 107 & 491 & $46.76 \%$ \\
\hline & Pobre & 58 & 40 & 45 & 69 & 64 & 47 & 323 & $30.76 \%$ \\
\hline & $\begin{array}{l}\text { Extrema } \\
\text { Pobreza }\end{array}$ & 41 & 42 & 58 & 39 & 35 & 21 & 236 & $22.48 \%$ \\
\hline \multirow{8}{*}{ Religión } & Católico & 105 & 101 & 103 & 83 & 67 & 96 & 555 & $52.86 \%$ \\
\hline & Evangélico & 63 & 72 & 67 & 77 & 90 & 66 & 435 & $41.43 \%$ \\
\hline & Agnóstico & 0 & 0 & 0 & 0 & 4 & 4 & 8 & $0.76 \%$ \\
\hline & Testigo de & & & & & & & & \\
\hline & Jehová & 1 & 1 & 0 & 1 & 2 & 2 & 7 & $0.67 \%$ \\
\hline & Adventista & 3 & 0 & 0 & 0 & 1 & 2 & 6 & $0.57 \%$ \\
\hline & Ateo & 1 & 0 & 0 & 1 & 0 & 1 & 3 & $0.29 \%$ \\
\hline & Otras & 2 & 1 & 5 & 13 & 11 & 4 & 36 & $3.43 \%$ \\
\hline Total & & 175 & 175 & 175 & 175 & 175 & 175 & 1,050 & $100 \%$ \\
\hline
\end{tabular}

*Según clasificación de las Necesidades Básicas Insatisfechas del Instituto Nacional de Estadística (INE).

Rev. cienc. forenses Honduras, Volumen 7, № 1, Año 2021

Open Access

(c)Autor(es).
URL: http://www.bvs.hn/RCFH/html5/ CC-BY-NC 4.0 internacional 


\begin{tabular}{|c|c|c|c|c|}
\hline \multicolumn{5}{|c|}{ Cuadro 2. Relación actitud donante según área de procedencia } \\
\hline Actitud donante & Comayagua & Intibucá & Francisco Morazán & Porcentaje global \\
\hline \multicolumn{5}{|c|}{ A (Positiva) } \\
\hline \multirow[t]{2}{*}{ Rural } & 154 & 92 & 134 & 379 \\
\hline & $44 \%$ & $26.3 \%$ & $38.3 \%$ & $36.1 \%$ \\
\hline \multirow[t]{2}{*}{ Urbano } & 151 & 137 & 119 & 407 \\
\hline & $43.1 \%$ & $\underline{38.9 \%}$ & $34 \%$ & $38.8 \%$ \\
\hline \multicolumn{5}{|c|}{ B (Negativa) } \\
\hline \multirow[t]{2}{*}{ Rural } & 21 & 83 & 41 & 146 \\
\hline & $6 \%$ & $23.7 \%$ & $11.7 \%$ & $13.9 \%$ \\
\hline \multirow[t]{2}{*}{ Urbana } & 24 & 39 & 56 & 118 \\
\hline & $6.9 \%$ & $11.1 \%$ & $16 \%$ & $11.2 \%$ \\
\hline Total & 350 & 350 & 350 & 1,050 \\
\hline
\end{tabular}

A: Actitud donante positiva. B: Actitud donante negativa por departamento con su frecuencia y porcentaje específico y global.

\begin{tabular}{|c|c|c|c|c|c|c|c|}
\hline \multicolumn{8}{|c|}{$\begin{array}{l}\text { Cuadro 3. Fundamentos principales de actitud negativa } \\
\text { según departamento }\end{array}$} \\
\hline & \multicolumn{2}{|c|}{ Comayagua } & \multicolumn{2}{|c|}{ Intibucá } & \multicolumn{2}{|c|}{$\begin{array}{l}\text { Francisco } \\
\text { Morazán }\end{array}$} & \multirow[b]{2}{*}{ Global ( $n$ y \%) } \\
\hline & Rural & Urbana & Rural & Urbana & Rural & Urbana & \\
\hline Otras ** & $\begin{array}{c}4 \\
8.9 \%\end{array}$ & $\begin{array}{c}8 \\
17.8 \%\end{array}$ & $\begin{array}{c}31 \\
25.4 \%\end{array}$ & $\begin{array}{c}19 \\
15.6 \%\end{array}$ & $\begin{array}{c}32 \\
33 \%\end{array}$ & $\begin{array}{c}23 \\
23.7 \%\end{array}$ & $\begin{array}{c}117 \\
44.3 \%\end{array}$ \\
\hline $\begin{array}{l}\text { Por la idea de } \\
\text { mutilación del } \\
\text { cuerpo }\end{array}$ & $\begin{array}{c}9 \\
20 \%\end{array}$ & $\begin{array}{c}9 \\
20 \%\end{array}$ & $\begin{array}{c}19 \\
15.6 \%\end{array}$ & $\begin{array}{c}18 \\
14.8 \%\end{array}$ & $\begin{array}{c}7 \\
7.2 \%\end{array}$ & $\begin{array}{c}8 \\
8.2 \%\end{array}$ & $\begin{array}{c}70 \\
26.5 \%\end{array}$ \\
\hline $\begin{array}{l}\text { Por motivos } \\
\text { religiosos }\end{array}$ & $\begin{array}{c}4 \\
8.9 \%\end{array}$ & $\begin{array}{c}5 \\
11,1 \%\end{array}$ & $\begin{array}{c}10 \\
8.2 \%\end{array}$ & $\begin{array}{c}5 \\
4.1 \%\end{array}$ & $\begin{array}{c}6 \\
6.2 \%\end{array}$ & $\begin{array}{c}10 \\
10.3 \%\end{array}$ & $\begin{array}{c}40 \\
15.2 \%\end{array}$ \\
\hline $\begin{array}{l}\text { Por miedo que } \\
\text { retiren los órganos } \\
\text { antes de estar } \\
\text { realmente muerto }\end{array}$ & $\begin{array}{c}5 \\
11.1 \%\end{array}$ & $\begin{array}{c}0 \\
0 \%\end{array}$ & $\begin{array}{c}13 \\
10.7 \%\end{array}$ & $\begin{array}{c}3 \\
2.5 \%\end{array}$ & $\begin{array}{c}6 \\
6.2 \%\end{array}$ & $\begin{array}{c}5 \\
5.2 \%\end{array}$ & $\begin{array}{c}32 \\
12.1 \%\end{array}$ \\
\hline No pagan dinero & $\begin{array}{c}1 \\
2.2 \%\end{array}$ & $\begin{array}{c}0 \\
0 \%\end{array}$ & $\begin{array}{c}4 \\
3.3 \%\end{array}$ & $\begin{array}{c}0 \\
0 \%\end{array}$ & $\begin{array}{c}0 \\
0 \%\end{array}$ & $\begin{array}{c}0 \\
0 \%\end{array}$ & $\begin{array}{c}5 \\
1.9 \%\end{array}$ \\
\hline$n$ total & 23 & 22 & 77 & 45 & 51 & 46 & 264 \\
\hline
\end{tabular}

Rev. cienc. forenses Honduras, Volumen 7, № 1, Año 2021

Open Access

(c) Autor(es).
URL: http://www.bvs.hn/RCFH/html5/ CC-BY-NC 4.0 internacional 
conocimiento entre el área rural y la urbana. En Francisco Morazán, en las cuatro variables evaluadas, se observó menor conocimiento en el área rural en comparación a con la urbana; en Intibucá en la variable "qué es la córnea" y coincidiendo este mismo hallazgo en los tres departamentos acerca de las características requeridas para ser donante. La comparación del porcentaje de conocimientos respecto a las variables estudiadas por departamento y área de procedencia se detalla en el cuadro 4.

\section{Relación actitud donante-conocimiento}

Los participantes con mayor nivel de conocimiento respecto a las variables evaluadas, mostraron mayor disposición a donar en los tres departamentos. Se observó que la actitud donante positiva es proporcional al conocimiento. De los participantes que no conocían del tema, el 36\% (106/229) presentó una actitud negativa hacia la donación., a diferencia de los participantes que conocían, lo suficiente, únicamente el $16 \%$ (24/148) presentó una actitud negativa. Este resultado se obtuvo en el área urbana tanto en Comayagua $\left(p=0.026, x^{2}=9.3031\right)$, Francisco Morazán $\left(p=0.0001 x^{2}=18.1563\right)$ e Intibucá $(p=0.0252$, $\left.x^{2}=9.1236\right)$. En el área rural, la disposición a donar no dependía del conocimiento. Respecto a los conocimientos relacionados al trasplante de córnea, en Intibucá el 39\% (99/254) de los participantes que desconocían qué es la córnea estuvo en contra de la donación, en contraste con los que sabían lo suficiente donde únicamente el $4.7 \%(1 / 21)$ estuvo en contra de ello., tanto en el área urbana como rural $\left(p=0.0062 x^{2}=10.8438\right)$. Relación similar en la variable de realización de trasplante de córnea y características del donante en Francisco Morazán, Comayagua e Intibucá, como se detallada en el cuadro 5.

El 86.67\% $(910 / 1,050)$ de la población de todos los departamentos, indistintamente de su procedencia, manifestó el deseo de recibir trasplante de córnea de ser necesario, únicamente el $13.33 \%$ no lo recibirían. En Comayagua, $7.14 \%$ mostro una actitud receptora negativa, de estos el $40 \%$ consideró que, al realizar el procedimiento, su rostro quedaría deforme. En Intibucá el 18.29\% estuvo en desacuerdo a recibir trasplante el principal motivo fue desconocer la reacción de la familia en un $37.14 \%$. En Francisco Morazán el 14.57\% de los cuales el $26.32 \%$ manifestó como principal causa de rechazo otras razones privadas.

\section{DISCUSIÓN}

Pese a que en Honduras se cuenta con los eslabones pertinentes para que se realicen trasplantes a partir de donante cadavérico, 


\begin{tabular}{|c|c|c|c|c|c|c|}
\hline \multicolumn{7}{|c|}{$\begin{array}{l}\text { Cuadro 4. Resumen de conocimiento sobre donación y trasplante de córnea por departamento y área de } \\
\text { procedencia }\end{array}$} \\
\hline Conocimiento & \multicolumn{2}{|c|}{ Comayagua } & \multicolumn{2}{|c|}{ Intibucá } & \multicolumn{2}{|c|}{ Francisco Morazán } \\
\hline \multicolumn{7}{|c|}{ Donación de órganos } \\
\hline Nivel & $\begin{array}{l}\text { Rural } \\
n(\%)\end{array}$ & $\begin{array}{c}\text { Urbana } \\
n(\%)\end{array}$ & $\begin{array}{l}\text { Rural } \\
n(\%)\end{array}$ & $\begin{array}{c}\text { Urbana } \\
n(\%)\end{array}$ & $\begin{array}{l}\text { Rural } \\
N(\%)\end{array}$ & $\begin{array}{c}\text { Urbana } \\
n(\%)\end{array}$ \\
\hline Nada & $47(27)$ & $42(24)$ & $92(53)$ & $47(27)$ & $37(21)$ & $34(19)$ \\
\hline Poco y muy poco & $110(63)$ & $117(67)$ & 69(39) & $99(57)$ & $112(64)$ & $96(55)$ \\
\hline Lo suficiente & $18(10)$ & $16(9)$ & $14(8)$ & $29(17)$ & $26(15)$ & $45(26)$ \\
\hline$P y X^{2}$ & \multicolumn{2}{|c|}{$\begin{array}{c}P=0.0039 \\
X 2=12.1611 p\end{array}$} & \multicolumn{2}{|c|}{$p=0$} & \multicolumn{2}{|c|}{$\begin{array}{l}p=0.0431 \\
X^{2}=8.1458\end{array}$} \\
\hline \multicolumn{7}{|l|}{ Que es la córnea } \\
\hline Nada & $94(54)$ & $74(42)$ & $144(82)$ & $110(63)$ & $123(70)$ & $46(26)$ \\
\hline Poco y muy poco & 69(39) & $88(50)$ & $26(15)$ & $49(28)$ & $44(25)$ & $87(50)$ \\
\hline Lo suficiente & $12(7)$ & $13(7)$ & $5(3)$ & $16(9)$ & $8(5)$ & $42(24)$ \\
\hline$P$ y $X^{2}$ & \multicolumn{2}{|c|}{$\begin{aligned} P & =0.3472 \\
X 2 & =3.5576\end{aligned}$} & \multicolumn{2}{|c|}{$\begin{aligned} p & =0.0006 \\
X^{2} & =17.3860\end{aligned}$} & \multicolumn{2}{|c|}{$\begin{array}{l}p=0.00001 \\
X^{2}=74.109\end{array}$} \\
\hline \multicolumn{7}{|c|}{ Realización de trasplantes de córnea en Honduras } \\
\hline Nada & 121(69) & $99(57)$ & $156(89)$ & $137(78)$ & $147(84)$ & $73(42)$ \\
\hline Poco y muy poco & $51(29)$ & 69(39) & 16(9) & $32(18)$ & $22(13)$ & $77(44)$ \\
\hline Lo suficiente & $3(2)$ & $7(4)$ & $3(2)$ & $6(3)$ & $6(3)$ & $25(14)$ \\
\hline PY $X^{2}$ & \multicolumn{2}{|c|}{$\begin{array}{c}P=0.6992 \\
X 2=1.6654\end{array}$} & \multicolumn{2}{|c|}{$\begin{aligned} P & =0.829 \\
X 2 & =0.7361\end{aligned}$} & \multicolumn{2}{|c|}{$\begin{array}{l}p=0.00001 \\
X^{2}=67.8997\end{array}$} \\
\hline \multicolumn{7}{|c|}{ Características del donante } \\
\hline Nada & $86(49)$ & $62(35)$ & $127(73)$ & $92(53)$ & $82(47)$ & $96(55)$ \\
\hline Lo suficiente & $89(51)$ & $113(65)$ & $48(27)$ & $83(47)$ & $93(53)$ & $79(45)$ \\
\hline PY $X^{2}$ & \multicolumn{2}{|c|}{$\begin{array}{l}p=0.0271 \\
X^{2}=7.2151\end{array}$} & \multicolumn{2}{|c|}{$\begin{aligned} p & =0.0005 \\
X^{2} & =15.3786\end{aligned}$} & \multicolumn{2}{|c|}{$\begin{array}{l}p<0.00001 \\
X^{2}=20.8965\end{array}$} \\
\hline
\end{tabular}

aún no se cuenta con un banco de ojos, ni se realiza el proceso de donación cadavérica ${ }^{8}$.

En el 2017 aproximadamente 600 personas urgían de un trasplante de córnea en el país. El único centro asistencial a nivel público que realiza este trasplante es el Hospital San Felipe, ubicado en Tegucigalpa, la capital de Honduras. El costo para los pacientes que se someten a este procedimiento es de 2000 lempiras (80 dólares aproximadamente, que cubre únicamente el transporte de las córneas de Estados Unidos a Honduras); se realizan aproximadamente doce trasplantes mensuales, lo cual es posible gracias a un convenio que se realizó con Sight life (https://sightlife.org/), organización que de manera 


\begin{tabular}{|c|c|c|c|c|c|c|}
\hline \multicolumn{7}{|c|}{ Cuadro 5. Relación estadística de la actitud donante en relación al conocimiento } \\
\hline Conocimiento & \multicolumn{2}{|c|}{ Comayagua } & \multicolumn{2}{|c|}{ Intibucá } & \multicolumn{2}{|c|}{ Francisco .Morazán . } \\
\hline Nivel & \multicolumn{6}{|c|}{ Actitud Donante } \\
\hline \multicolumn{7}{|c|}{ Donación de órganos } \\
\hline & Positiva & Negativa & Positiva & Negativa & Positiva & Negativa \\
\hline Nada & 71 & 18 & 75 & 64 & 47 & 24 \\
\hline Poco y muy poco & 207 & 20 & 118 & 50 & 143 & 65 \\
\hline Lo suficiente & 27 & 7 & 34 & 9 & 63 & 8 \\
\hline$P y X^{2}$ & \multicolumn{2}{|c|}{$\begin{array}{c}p=0.039 \\
X^{2}=12.1611\end{array}$} & \multicolumn{2}{|c|}{$\begin{aligned} p & =0.0019 \\
X^{2} & =14.9554\end{aligned}$} & \multicolumn{2}{|c|}{$\begin{array}{c}p=0.0012 \\
X^{2}=14.6123\end{array}$} \\
\hline \multicolumn{7}{|l|}{ Que es la córnea } \\
\hline Nada & 141 & 27 & 155 & 99 & 111 & 58 \\
\hline Poco y muy poco & 141 & 16 & 52 & 23 & 95 & 36 \\
\hline Lo suficiente & 23 & 2 & 20 & 1 & 47 & 3 \\
\hline$p y X^{2}$ & \multicolumn{2}{|c|}{$\begin{array}{c}P=0.21 \\
X 2=4.5042\end{array}$} & \multicolumn{2}{|c|}{$\begin{array}{c}p=0.0062 \\
X^{2}=10.8438\end{array}$} & \multicolumn{2}{|c|}{$P=<0.00001$} \\
\hline \multicolumn{7}{|c|}{ Realización de trasplantes de córnea en Honduras } \\
\hline Nada & 188 & 32 & 183 & 110 & 149 & 71 \\
\hline Poco y muy poco & 108 & 12 & 38 & 10 & 74 & 25 \\
\hline Lo suficiente & 9 & 1 & 6 & 3 & 30 & 1 \\
\hline$p y X^{2}$ & \multicolumn{2}{|c|}{$\begin{array}{c}P=0.6923 \\
X 2=1.4568\end{array}$} & \multicolumn{2}{|c|}{$\begin{array}{c}P=0.102 \\
X 2=5.9884\end{array}$} & \multicolumn{2}{|c|}{$\begin{array}{l}P=0.0391 \\
X^{2}=8.3618\end{array}$} \\
\hline \multicolumn{7}{|c|}{ Características el donante } \\
\hline Nada & 121 & 27 & 114 & 105 & 100 & 78 \\
\hline Lo suficiente & 184 & 18 & 113 & 18 & 153 & 19 \\
\hline$p y X^{2}$ & \multicolumn{2}{|c|}{$\begin{array}{c}P=0.008 \\
X^{2}=6.6208\end{array}$} & \multicolumn{2}{|c|}{$\begin{array}{l}P<0.00001 \\
X^{2}=41.9549\end{array}$} & \multicolumn{2}{|c|}{$P=<0.000001$} \\
\hline
\end{tabular}

gratuita dona las córneas a nuestro país. En Centroamérica los países que cuentan con banco de córneas son Guatemala, Costa Rica y Panamá ${ }^{19}$.

Los resultados obtenidos en este estudio indican que, en general, la población encuestada presenta una actitud positiva a donar (74.86\%) y a recibir trasplantes $(86,87 \%)$, resultados que concuerdan con el estudio previamente realizado en nuestro país en el año 2017 , en el cual el $70 \%$ de los participantes manifestó estar dispuesto a donar sus órganos y/o tejidos cuando muera ${ }^{8}$. 
La relación descriptiva de la actitud donante y el compromiso de respaldo en su decisión según área de procedencia, es disímil ya que, tanto en el área rural como urbana, el porcentaje de aceptación de ser portador de un carnet que lo acredite como donante es del $48.5 \%$ y de estar registrado en un listado es del 59.2\%;porcentajes menores, en comparación a los resultados obtenidos en la actitud donante positiva (74.86\%), resultados que se asemejan a lo encontrado en un estudio realizado en España por la Coordinación Regional de Trasplantes de la Comunidad Autónoma de Murcia en el que la opinión hacia la donación de órganos sólidos propios fue favorable en el $63 \%$ de los encuestados $(n=1274)$, pero únicamente un $11 \%$ de ellos contaban con el carnet de donante ${ }^{20}$.

En los departamentos de Comayagua y Francisco Morazán, el análisis de los datos no sugiere que la procedencia rural o urbana, incida en la actitud positiva frente a la donación, $\left(x^{2}=3.2089, \quad P=0.0943\right)$ y $\left(x^{2}=0.8733, p=0.99\right)$ respectivamente; hallazgos similares se encontraron en España, donde la distribución geográfica no fue un factor influyente en la disposición a donar ${ }^{20}$. No fue así en el departamento de Intibucá $\left(x^{2}=24.29\right.$, $p=0.0005)$, donde la procedencia, rural o urbana, sí marcó una diferencia en la actitud hacia la donación, lo que podría estar asociado al nivel socio-económico y educativo de la población de este departamento, ya que este presenta mayores porcentajes de pobreza (61.7\%) y menor escolaridad (72\% en el área rural); hallazgos similares se encontraron en Arabia Saudita, donde observaron diferencias en la disposición a donar entre las poblaciones rurales y urbanas ${ }^{21}$.

Al evaluar el nivel de conocimiento se observó que los participantes del área urbana que conocían lo suficiente de los factores evaluados, presentaron menores porcentajes de actitud negativa hacia la donación, en contraste con los que no conocían nada, quienes reportaron porcentajes negativos mayores. En un estudio del 2001, en España, Martínez, López y Martin concluyeron que: "el grado de información y el conocimiento de los diferentes aspectos relacionados con el trasplante parecen tener también una importante influencia sobre la postura personal hacia la donación. De esta forma, se ha mostrado que un mayor nivel de conocimientos sobre la necesidad y los procedimientos del trasplante y la donación, se asocia a una disposición más favorable" ${ }^{22}$. En Emiratos Árabes Unidos, AlHajri, AlHebsi y AlSuwaidi obtuvieron resultados similares y concluyeron: "El hecho de no tener una comprensión global de la donación de órganos 
debido a la falta de conocimientos parece influir en la toma de decisiones, la mayoría de los participantes pensaba que la donación de órganos no les afectaba directamente lo que es un claro indicio de que la concientización es crucial" $^{\prime 4}$.

En relación a la situación laboral y parentela (hijos o no) solamente se encontró relevancia significativa en el departamento de Intibucá. Las personas que presentaron una actitud negativa en el área rural estaban desempleadas en el área rural ( $p=0.0164, O R=1.95, I C=1.24-3.08)$; y además tenían descendencia tanto en el área urbana como rural $(P=0.023, O R=0.33, I C=0.17$ 0.64); al igual en un estudio realizado en Perú donde se encontró que se asociaron de manera significativa tener un empleo con una actitud positiva (18.37\%). ${ }^{29}$ y en un estudio en España en donde se asoció la descendencia como un factor negativo. (70\% versus $60 \% ; p=0,001)^{20}$.

Respecto a la religión pese a que ninguna religión prohíbe explícitamente la donación de $\operatorname{organos}^{26,27}$, encontramos en nuestro estudio grupos religiosos minoritarios que se oponen a la donación; en Comayagua, en el área rural, los practicantes Testigos de Jehová y adventistas están contra de la donación. $\left(X^{2}=26.83\right.$, $p=0.0001$ ), observando el mismo resultado estadístico en el área urbana de Francisco
Morazán en el área urbana. $\left(X^{2}=35.47\right.$, $p=0.00001$ ), esto similar al estudio realizado en Arabia Saudita en donde la población del área rural expresó altos porcentajes de actitud negativa por motivos religiosos en comparación al área urbana, sin embargo, no fueron estadísticamente significativos ${ }^{21}$.

Los resultados de este estudio indican un porcentaje positivo hacia la donación en general. Principalmente en los departamentos más desarrollados como ser Francisco Morazán y Comayagua. Sin embargo, existe un porcentaje de los participantes que no están a favor de la donación. La desinformación es una de las principales limitantes, ya que estadísticamente existe relación entre el conocimiento y la actitud donante. Sin embargo, las causas de rechazo no fueron claras en su totalidad. Muchos de los participantes mostraban indiferencia hacia el tema incluyendo los que están a favor, pero, predominantemente en el área rural, rechazan la idea de obtener un documento que le respalde su decisión, predominantemente en el área rural.

Para realizar una inversión como el banco de ojos en Honduras es importante superar las limitaciones impuestas por la desinformación tanto en el área urbana como rural. A pesar de 
los mayores niveles de conocimiento obtenidos en el área urbana, aún existen temas relevantes a la donación importantes de aclarar y definir. principalmente establecer los conceptos generales, las características del donante, los conceptos médicos y legales necesarios y sobre todo promover y fomentar la solidaridad $\mathrm{y}$ reciprocidad. Para ello es necesario impulsar una cultura de donación a través de la creación de estrategias comunicativas de educación que consideren los factores propios de los grupos poblacionales de nuestro país.

\section{Agradecimientos}

Agradecemos a la Dra. Mireya Matamoros por su dedicación y ayuda en la elaboración del manuscrito.

\section{REFERENCIAS BIBLIOGRÁFICAS}

1. Organización Panamericana de la Salud. Ministros de Salud acuerdan plan para aumentar la donación de órganos y los trasplantes, que son insuficientes para la demanda. [Internet] Washington, DC. 2019 [citado el 11 de octubre del 2019] Disponible en:

https://www.paho.org/hq/index.php?option=c om content\&view=article\&id=15487: ministers -of-health-agree-on-plan-to-increase-organdonations-and-transplants-in-an-effort-to$\underline{\text { meet-demand-2\&ltemid=1926\&lang=es }}$
2. Viteri N. Factores sociodemográficos y culturales de los pacientes trasplantados de córnea en el sistema nacional de salud. 20122016. Pontificia Universidad Católica Del Ecuador, Facultad de Medicina [Monografía en línea] Quito, Ecuador 2018[citado el 11 de octubre del 2019] disponible en: http://repositorio.puce.edu.ec/bitstream/hand le/22000/15224/FACTORES\%20SOCIODEMOGR AFICOS\%20Y\%20CULTURALES\%20DE\%20LOS\% 20PACIENTES\%20TRASPLANTADOS\%20DE\%20 CORNEA\%20EN\%20EL\%20SISTEMA\%20NA.pdf? sequence $=1 \&$ isAllowed $=y$

3. Zamora A, Díaz Y. Factores que inciden en la cultura de donación de órganos en Morelia, Michoacán: hacia una propuesta de política pública. Tla-melaua [internet]. 2018[citado el 22/4/21], vol.12, n.44, pp.98119.

Disponible en:

http://www.scielo.org.mx/scielo.php?pid=S187 $\underline{0-69162018000100098 \& \text { script=sci abstract }}$

4. AlHajri L, AlHebsi A, AlSuwaidi M. Cómo el contexto afecta la disposición de las personas a registrarse en el programa de donación de órganos de fallecidos. Salud Pública de BMC. [internet]2021 [citado 22/4/21]; 21 (1): 729. Disponible en: https://bmcpublichealth.biomedcentral.com/a rticles/10.1186/s12889-021-10753-5
Rev. cienc. forenses Honduras, Volumen 7, № 1, Año 2021 Open Access (c)Autor(es).
URL: http://www.bvs.hn/RCFH/html5/ CC-BY-NC 4.0 internacional 
5. Montero A, Martínez S, Fieira E, Fernández A, Castro D, Fernández D. Conocimientos y actitudes ante la donación de órganos de los profesionales sanitarios de un hospital de tercer nivel. Rev. Esp. Salud Pública [Internet]. 2018 [citado 12 de octubre de 2019]; vol 92: e201804007. Disponible en: http://scielo.isciii.es/scielo.php?script=sci artt ext\&pid=S1135-57272018000100205\&lng=es.

6. Tello I. Actitudes y conocimientos frente a la donación de órganos y tejidos. CASUS [Internet] 2017 [citado el 11 de octubre del 2019]; vol2(2):82-88. Disponible en: https://dialnet.unirioja.es/servlet/articulo?codi go $=6258754$

7. Sebastián M, Guerra E, Varga A, Barboza O, Ríos A. Actitud y conocimiento sobre donación de órganos de estudiantes de medicina de una universidad pública del noreste de México. Pubmed [artículo de revista en línea] GacMedMex. 2017 [citado el 11 de octubre del 2019]; 153:430-440 disponible en: https://www.anmm.org.mx/GMM/2017/n4/G MM 153 $2017 \quad 4$ 430-440.pdf

8. Matamoros $\mathrm{M}$, Castillo $\mathrm{B}$, Alvarado $\mathrm{D}$. Conocimientos, actitudes y creencias sobre donación y trasplante en Honduras. Rev. cienc. forenses Honduras [revista en la internet]. 2017; 3(1): 2-9. disponible en: http://www.bvs.hn/RCFH/pdf/2017/pdf/RCFH 3-1-2017-3.pdf
9. Ley de donación y trasplante de órganos anatómicos en seres humanos. La Gaceta No. 33412. Decreto 329-2013 26 de abril del 2014.

10. Castillo B, Matamoros M, Alger J, Alvarado D. Evaluación de la respuesta global de los procesos pre-trasplante de córneas cadavéricas en Medicina Forense de Tegucigalpa. Rev. Cienc. Forenses Honduras [Internet]. 2019 [Consultado 24 Jul 2019]; 5(1):3-12. Disponible en: https://www.lamjol.info/index.php/RCFH/artic le/view/8673

11. Espinal J, Cedillo M. Actuación del médico forense en la donación y trasplante de órganos. Revisión bibliográfica. Rev. cienc. forenses Honduras. 2017; 3(1): 26-39. [citado 25 de septiembre de 2019]. Disponible en: https://www.lamjol.info/index.php/RCFH/artic le/view/9817/11226

12. Aspectos generales de la comunidad, ubicación de la ciudad de Siguatepeque. [sede web] Capítulo VI pág. 6-11. [acceso 16 octubre 2019] disponible en: http://www.bvs.hn/TMH/pdf/TMH139/pdf/TM $\underline{\text { H139-5.pdf }}$

13. Dirección y Registro Catastro, Instituto de la Propiedad. Siguatepeque, Comayagua 2010-2019.

14. Informe Equipo de Salud Familiar ESFAM Aldea El Porvenir, Siguatepeque, Comayagua 2019. 
15. Jesús de Otoro [internet]. RedHonduras.com 2019 [citado el 6 de octubre del 2019]. [Citado 28 de septiembre de 2019]. Disponible

en: https://redhonduras.com/geografia/jesus-deotoro/

16. Instituto Nacional de Estadística. XVII Censo de Población y VI de vivienda 2013 [Internet].2013 [citado 03 de septiembre de 2019]. Disponible en: https://www.ine.gob.hn/images/Productos\%2 Oine/censo/Tomo\%20municipales \%20pdf/08FcoMorazán/110DistritoCentral.pdf 17. NU. CEAPL. División de Estadística y Proyecciones Económicas, 2001. El método de las necesidades básicas insatisfechas (NBI) y sus aplicaciones en América Latina. Santiago de Chile: CEPAL [citado 20/03/21], pp.11-19,51. Disponible en: https://repositorio.cepal.org/bitstream/handle L11362/4784/S0102117 es.pdf?sequence=1

18. CDC. (2019). Epi InfoTM (versión 7.2.3.1) [Windows]. Estados Unidos.

19. Sánchez R. Unas 600 personas urgen de trasplante de Córnea en el país. Diario El Heraldo. 2017, abril 23. País. 16(3).

20. Conesa C, Ríos A, Ramírez P. Estudio multivariado de los factores psicosociales que afectan la actitud del público hacia la donación de órganos. Nefrología: Publicación Oficial de la Sociedad Española Nefrología. [internet] 2005 [citado el 22/4/2021]; 25 (6): 684-697. Disponible

en:

https://www.revistanefrologia.com/es-

estudio-multivariante-de-los-factores-articulo-

\section{$\underline{X 0211699505019359}$}

21. Alghanim SA. Conocimientos y actitudes hacia la donación de órganos: un estudio comunitario que compara poblaciones rurales y urbanas. Saudi J Kidney DisTranspl [internet] 2010 [citado 22/4/20211]; 21: 23-30. Disponible en:

https://www.sjkdt.org/text.asp?2010/21/1/23 $\lcm{58703}$

22. López JS., Martín A., Martínez JM. Percepción social de la donación en España tras la década de los trasplantes. Nefrología[internet] 2001 [citado: 22/4/2021].21(S4):45-48. Disponible en: https://www.revistanefrologia.com/espercepcion-social-donacion-espana-trasarticulo-X0211699501027194

23. Ríos A, López A, García J, et al. The attitude of Latin American immigrants in Florida (USA) towards deceased organ donation - a cross section cohort study. Transplant. [internet] 2017 Oct [citado 22/4/21];30(10):1020-1031 disponible en: https://pubmed.ncbi.nlm.nih.gov/28608574/

24. Castillo M, Belén L, Arguello Z, Daniel J. Conocimiento y Predisposición Frente a la Donación de Órganos en Universitarios. XXVI JI 
Jornada de jóvenes investigadores AUGM 2018;

17-19 octubre; Argentina. Disponible en: https://bdigital.uncu.edu.ar/objetos digitales/ $\underline{12866 / 30-d o n a c i o n e s y t r a s p l a n t e s-m e r e l e s-~}$

liza-uni.pdf

25. Breitkopf CR. Actitudes, creencias y comportamientos en torno a la donación de órganos entre mujeres hispanas. Trasplante de órganos CurrOpin [internet]. 2009[citado 22/4/2021]; 14 (2): 191-195.Disponible en: https://www.ncbi.nlm.nih.gov/pmc/articles/P MC2687523/

26. Revello R. Donación de órganos y religiones. Vida y Ética. 2010(2) [citado 13 de octubre de 2019]. Disponible en: https://repositorio.uca.edu.ar/bitstream/1234 56789/1502/1/donacion-organosreligiones.pdf http://bibliotecavirtual.dgb. umich.mx:8083/xmlui/handl e/DGB UMICH/2439
27. Bruzzone P. Religious aspects of organ transplantation. Transplant Proc [internet] 2008 May [citado 22/4/2021]; 40(4):1064-7. Disponible en: https://pubmed.ncbi.nlm.nih.gov/18555116/ 28. Castañeda D, Alarcón F, Ovalle D, Martínez C, González L, Burbano L, León K, López L, Yaya J, Márquez L. Actitudes y creencias sobre la donación de órganos en Colombia: ¿Dónde se deben enfocar los esfuerzos para mejorar las tasas nacionales de donación?. rev.fac.med. [Internet]. Enero 2014 [citado 24/04/2021]; 62 ( 1 ): 17-25. Disponible en: http://www.scielo.org.co/pdf/rfmun/v62n1/v6 $\underline{2 n 1 a 03 . p d f}$ 